\title{
Free Vibration of Nanotubes Affected by Atomic Dislocation
}

\author{
Seckin Filiz \\ Tekirdag Namik Kemal University, Corlu Vocational School \\ 59860, Corlu, Turkey \\ sfiliz@nku.edu.tr
}

\begin{abstract}
In recent years, the invention of nanotubes has begun to find its own use area. Computer related researches have been developed for the first time in the world a logical computer circuit in a single molecule. Carbon atoms formed, hair from a tube-shaped molecule of 100,000 times thinner than the hair, carbon nanotubes produced the "voltage Inverter", one of the three basic circuits found on today's computers. In addition, the new generation transistor design is used in electronics. In terms of mechanical properties, the elasticity modulus is in the order of TPa. By the using of nano strain gauge devices, the mechanical properties of the material measurements will be more accurate. In this paper, nanobeam is formulated as an Euler-Bernoulli beam and the equation of motion will be solved by using central finite differences method.

By the invention of carbon nanotubes by Iijima [1], science has gained a new perspective. In the name of this perspective, nanomaterial is not so wrong. Nano scale materials have good electrical and mechanical properties. Thus, they can be applied for nanodevices and nanocomposites. After 1960s, Eringen concentrated its work on the mechanics of continuous materials and pioneered this branch of science. In this way, the effects of the borders between atoms are better understood [2-4].In 2009, Abbaschian and Reed-Hill examined the formation of dislocation between atoms, in detail [5].Wang and Varadan studied the behavior of free vibration in the swcnt Timoshenko beam using nonlocal elasticity in 2006 [6].

In this study, affected Single Walled Carbon Nanotubes (SWCNTs) will be studied using transverse vibration of CNTs in the framework of the Nonlocal Elasticity theory. Equations of motion of carbon nanotubes, numerical results are obtained for affected CNTs by using Euler Bernoulli beam theory. The effects of the first mode frequency parameter of SWCNT has affected (missing) mass are studied by using Central Finite Differences Method. The location of missing mass and nonlocal parameters on vibration of SWCNT is investigated.Simply Supported boundary condition is considered. The purpose of this study is to fill this gap by investigating vibration problems of carbon nanotubes have missing mass.
\end{abstract}

Keywords: Carbon Nanotubes, Euler Bernoulli Beam, Finite Differences Method, Vibration.

\section{Theory and Results}

A carbon nanotube of length L, coordinate system and carbon atom formation are shown in Fig.1. It is assumed that the material property is isotropic form.

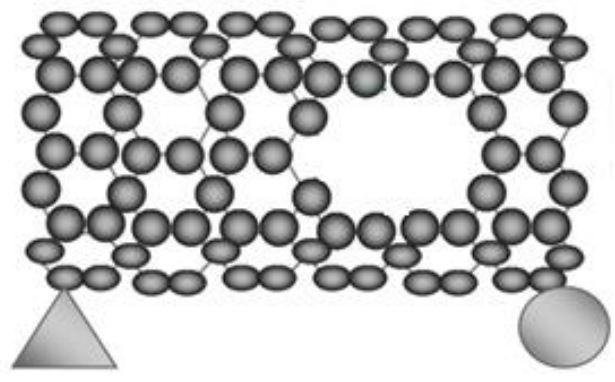

Fig. 1: Simply supported affected CNT.

The property of CNT is assumed to be in the composite structure. Since affected place leads to a variable coefficient differential equation for vibration of beam. 


$$
\sigma=E \varepsilon
$$

where, $\mathrm{E}$ is the elasticity modulus, $\sigma$ is stress and $\varepsilon$ is the strain. By using Hooke's Law (Equ. 1), the transverse vibration of carbon nanotube can be written in the following form:

$$
\left(1-\mu \frac{\partial^{2}}{\partial x^{2}}\right) \tau_{x x}=m \frac{\partial^{2} w}{\partial t^{2}}
$$

where, $\mathrm{w}(\mathrm{x}, \mathrm{y})$ is the displacement, $\mathrm{m}$ is the mass per unit length and $\tau_{\mathrm{xx}}$ is the nonlocal stress tensor and $\mu$ is the nonlocal elasticity constant. The equation of motion for the tube can be obtained as:

$$
\frac{\partial^{2} w}{\partial x^{2}}\left(E I \frac{\partial^{2} w}{\partial x^{2}}\right) \tau_{x x}=m \frac{\partial^{2} w}{\partial t^{2}}
$$

Using Eq. (3) free-vibrating CNT in the nonlocal elasticity can be found in terms of displacement:

$$
w(x, t)=W(x) e^{i \omega t}
$$

where, $\omega$ is angular frequency, $\mathrm{t}$ is time, $\mathrm{W}(\mathrm{x})$ is amplitude and $\mathrm{i}$ is imaginary number $\sqrt{-1}$. The displacement field is applied into Equ. 3, the equation of motion of CNT is obtained as:

$$
\frac{\partial^{4} w}{\partial x^{4}}-\mu \lambda^{2} \frac{\partial^{2} w}{\partial x^{2}}+\lambda^{2} w=0
$$

where, $\lambda=\sqrt{\frac{m \omega^{2} L^{4}}{E I}}$ is nondimensional parameter.

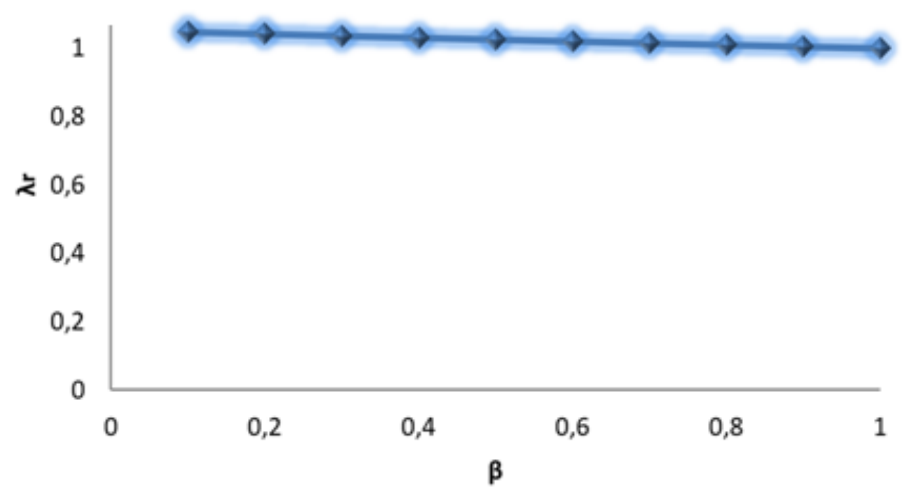

Fig. 2: Nondimensional fundamental frequency ratio $(\lambda r)$ for different $\beta$ of beam.

where $\beta$ refers to mass ratio and $\beta=\frac{m_{m}}{m_{T}} . \mathrm{m}_{\mathrm{m}}$ is ( $\mathrm{m}_{\mathrm{T}}-$ missing mass) and $\mathrm{m}_{\mathrm{T}}$ is total mass, In Fig. 2 , by increasing the $\beta$ rate, the frequency values are decreasing. So, the result can be achieved, increasing the value of the mass, the frequency values increase. where, $\lambda r$ is nondimensional frequency ratio and $\beta$ is mass ratio. 


\section{Discussions}

In this paper, on the transverse vibration of affected nanotube is studied. The material properties are isotropically changing in the thickness direction of the beam. The Euler-Bernoulli Beam Theory is used forsolving of the problem. Nondimensionalfrequencies were calculated according to the missing mass and the results of this solution obtained that frequencies were increasing using missing mass.

\section{References}

[1] S. Iijima, "Helical microtubules of graphitic carbon," Nature, vol. 354, p. 6348, 1991. DOI: 10.1038/354056a0.

[2] A. C. Eringen, "Nonlocal polar elastic continua," Int. J. Eng. Sci., vol. 10, pp. 1-16, 1972. DOI: 10.1016/00207225(72)90070-5.

[3] A. C. Eringen, "On differential equations of nonlocal elasticity and solutions of screw dislocation and surface waves," J. Appl. Phys., vol. 54, p. 4703, 1983. DOI: 10.1063/1.332803.

[4] A. C. Eringen, "Nonlocal Continuum Field Theories," Springer, VerlagPress, 2002.

[5] R. Abbaschian, R. E. Reed-Hill, "Physical Metallurgy Principles," Cengage Learning Publication, 2009.

[6] Q. Wang, V. K. Varadan, "Vibration of carbon nanotubes studied using nonlocal continuum mechanics," Smart Mater. Struct., vol. 15, pp. 659-666, 2006. DOI: 10.1088/0964-1726/15/2/050. 